\title{
HuConTest: Testing Human Contamination in Great Ape Samples
}

\author{
Martin Kuhlwilm $\mathbb{D}^{1, *}$, Claudia Fontsere $\mathbb{D}^{1}$, Sojung Han $\mathbb{D}^{1}$, Marina Alvarez-Estape $\mathbb{D}^{1}$, and \\ Tomas Marques-Bonet (D) $1,2,3,4$ \\ ${ }^{1}$ Institut de Biologia Evolutiva, CSIC-Universitat Pompeu Fabra, PRBB, Barcelona, Catalonia, Spain \\ ${ }^{2}$ CNAG-CRG, Centre for Genomic Regulation (CRG), Barcelona Institute of Science and Technology (BIST), Barcelona, Spain \\ ${ }^{3}$ Institucio Catalana de Recerca i Estudis Avançats (ICREA), Barcelona, Catalonia, Spain \\ ${ }^{4}$ Institut Català de Paleontologia Miquel Crusafont, Universitat Autònoma de Barcelona, Edifici ICTA-ICP, Cerdanyola del Vallès, Barcelona, Spain \\ *Corresponding author: E-mail: martin.kuhlwilm@upf.edu.
}

Accepted: 19 May 2021

\begin{abstract}
Modern human contamination is a common problem in ancient DNA studies. We provide evidence that this issue is also present in studies in great apes, which are our closest living relatives, for example in noninvasive samples. Here, we present a simple method to detect human contamination in short-read sequencing data from different species: HuConTest. We demonstrate its feasibility using blood and tissue samples from these species. This test is particularly useful for more complex samples (such as museum and noninvasive samples) which have smaller amounts of endogenous DNA, as we show here.
\end{abstract}

Key words: contamination, nonhuman primates, next-generation sequencing, fecal DNA, ancient DNA.

\section{Significance}

Human contamination can be a confounding factor in genomic studies, especially in the case of fecal, museum, or ancient DNA from great apes. It is important for quality assessment, screening purposes, and prioritization to identify and quantify such contamination. The tool presented here is a simple and versatile method for this purpose and can be applied to a wide range of sample types.

\section{Main Text}

Contaminationfrom exogenous sources is a problem common in ancient DNA, where multiple tools exist (reviewed by Peyrégne and Prüfer (2020)), as well as in studies of nonhuman primates (Prado-Martinez et al. 2013). Specifically, human contamination may occur in great ape samples of various origin and quality. Previously, differences in the mitochondrial genome between species were used to assess contamination (Prado-Martinez et al. 2013), which is a sensible strategy for high-coverage data. However, this approach is of limited use for shallow shotgun sequencing, especially of samples with low endogenous DNA content, such as fecal, historical, or ancient samples, as well as sequencing data obtained after enrichment through capture (Fontsere et al. 2020). Here, we devise a strategy based on diagnostic sites dispersed across the autosomes which can help detecting human contamination in an unbiased manner and with sparse data available.

\section{Determination of Diagnostic Sites}

We used previously published diversity data on high-coverage genomes from all great apes and modern humans (supplementary table S1, Supplementary Material online, fig. 1A), specifically, genomes from 58 chimpanzees and 10 bonobos (Pan clade) (Prado-Martinez et al. 2013; De Manuel et al. 2016), 43 gorillas (Gorilla clade) (Prado-Martinez et al. 2013; Xue et al. 2015), 27 orangutans (Pongo clade) (Prado-Martinez et al. 2013; Nater et al. 2017), and 19

(C) The Author(s) 2021. Published by Oxford University Press on behalf of the Society for Molecular Biology and Evolution.

This is an Open Access article distributed under the terms of the Creative Commons Attribution License (http://creativecommons.org/licenses/by/4.0/), which permits unrestricted reuse, distribution, and reproduction in any medium, provided the original work is properly cited. 
modern humans from Africa and western Eurasia (Mallick et al. 2016). The latter was chosen to represent the most common variation across modern humans. All genomes were processed as described previously (De Manuel et al. 2016): Sequencing data were mapped to the human genome (hg19) using BWA-MEM 0.7.7 (Li and Durbin 2009), PCR duplicates were removed using samtools (Li et al. 2009), and reads were locally realigned around indels using the GATK IndelRealigner 3.4-46 (McKenna et al. 2010). Genotypes were obtained individually using GATK UnifiedGenotyper with the EMIT_ALL_SITES parameter, and GVCFs from individuals were merged with GATK CombineVariants. The three species complexes Pan, Gorilla, and Pongo were then filtered separately: biallelic SNPs within each species complex together with humans were retrieved and filtered to exclude repetitive regions of the genome and regions with low mappability (35mer mappability). Finally, for each individual, genotypes were set to missing at sequencing coverage lower than 6 and higher than 100, and with a mapping quality lower than 20.

We retrieved SNPs where at least $98 \%$ of the chromosomes in the species complex showed the alternative allele (different from the human reference), with $<5 \%$ of missing genotypes, and where all modern human chromosomes included in this study carried the human reference allele, without allowing for missing genotypes. We allowed for residual amounts of human-reference-like genotypes in the great ape species, to account for residual sources of error in the reference set that might result in erroneous rare variation, and we deemed tolerating these a conservative strategy for determining diagnostic sites. Across the whole genome, we find 4,460,987 diagnostic sites for Pan species, 6,981,108 diagnostic sites for Gorilla species, and 7,518,570 diagnostic sites for Pongo species. The differences between species are partially explained by their evolutionary divergence to humans (Kaessmann and Pääbo 2002; Prado-Martinez et al. 2013; Kuhlwilm et al. 2016) but also the number of individuals used, as well as sequencing quality and coverage. We used the $\mathrm{R}$ package rtracklayer (Lawrence et al. 2009) to perform a liftover of these positions to the human genome version 38 (GRCh38).

\section{Contamination Assessment and Performance}

Contamination is defined here as the proportion of observed human reference alleles at diagnostic positions in short sequencing reads (fig. 1B). The test itself is wrapped in an $\mathrm{R}$ script (confirmed to work with $R$ versions 3.2.0, 3.4.4, 3.5.0, 3.6.0, and 4.0.1 (R Core Team 2015)), to directly process the number of reads carrying the reference or the alternative allele. We use samtools mpileup (tested for version 1.0 and 1.9) to obtain read depth and alternative read depth at diagnostic sites, and join these data with information on the alternative allele in the test species. We then calculate the number of reads matching the target species complex allele and subtract this value from the total read depth, thus retrieving the number of reads matching the human reference allele (more strictly, not matching the target species complex allele). We perform this calculation for each chromosome separately in order to obtain the standard variation and report the genomewide point estimate, one standard deviation, and the number of positions observed by the test. The latter value is useful to assess the reliability of the test at extremely shallow sequencing. The test can be applied to files with a bam or cram extension, containing short sequencing reads mapped to the human genome (hg19 or GRCh38). The basic filtering at this step can be simple, but it is advisable to remove adapter sequences (Schubert et al. 2016) and PCR duplicates to assess the unique contaminant fraction, as well as unmapped reads, nonprimary alignments, and sequences with a low mapping quality $(<30)$. We specifically recommend filtering the sequences on fragment/insert length ( $\geq 35$ base pairs) to avoid spurious alignments, which may happen at a high rate in the case of samples with large amounts of bacterial DNA (Meyer et al. 2016).

We tested the contamination test by artificially introducing modern human sequencing reads into bam files from the other species (in silico contamination), using eight human individuals that were not part of the reference panel (supplementary table S2, Supplementary Material online) (Auton et al. 2015), and great ape samples from other studies (Locke et al. 2011; Prüfer et al. 2012; Besenbacher et al. 2019). First, each human bam file was downsampled to $\sim 1.14 \mathrm{M}$ reads and merged with a chimpanzee bam file (ERR032960), to simulate $\sim 5 \%$ of human contamination. Since the read length differs between sequencing libraries from different studies, we account for the expected amount of human contamination by using the percentage of human base pairs added to the final bam file. After running the human contamination test in each file, we detect an average of $5.5 \%$ human contamination (supplementary table S2, Supplementary Material online), with minimal differences between humans from different world regions. When testing a gradient of increasing amounts of introduced human sequences from $\sim 0.1 \%$ to $\sim 39 \%$ to a chimpanzee bam file (supplementary table S3, Supplementary Material online, fig. 1C), the contamination is estimated correctly. The test is performing well for in silico contamination from modern humans in each of the great ape species (supplementary table S4, Supplementary Material online).

We also determined the inferred amount in the case of crosstesting, that is, performing the test of diagnostic sites for one clade in samples from the other clades (supplementary table S5, Supplementary Material online). Practically, this could be the relevant when, for example, a fecal sample was attributed to a chimpanzee but originated from a gorilla. We find contamination estimates of $44-82 \%$, depending on 
A

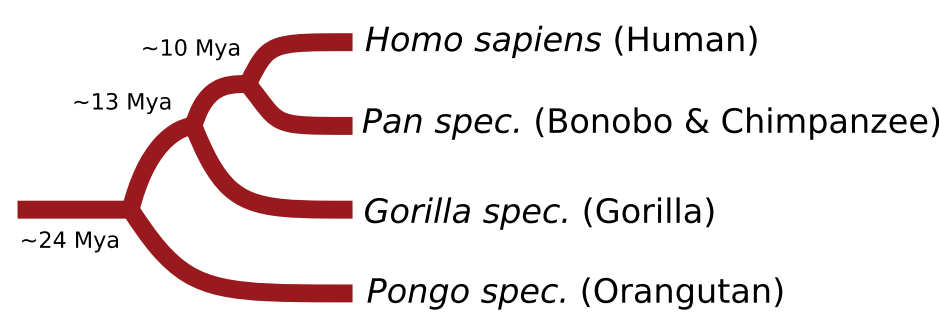

B

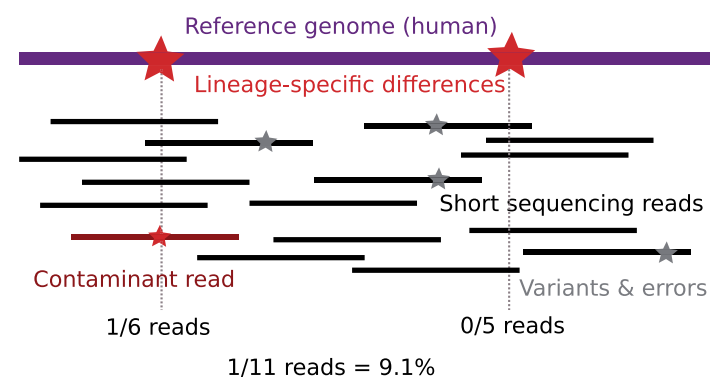

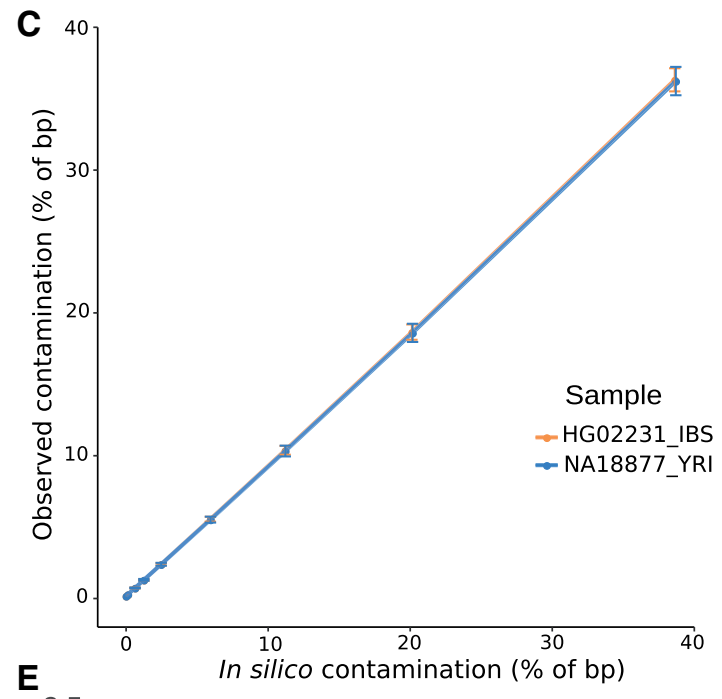
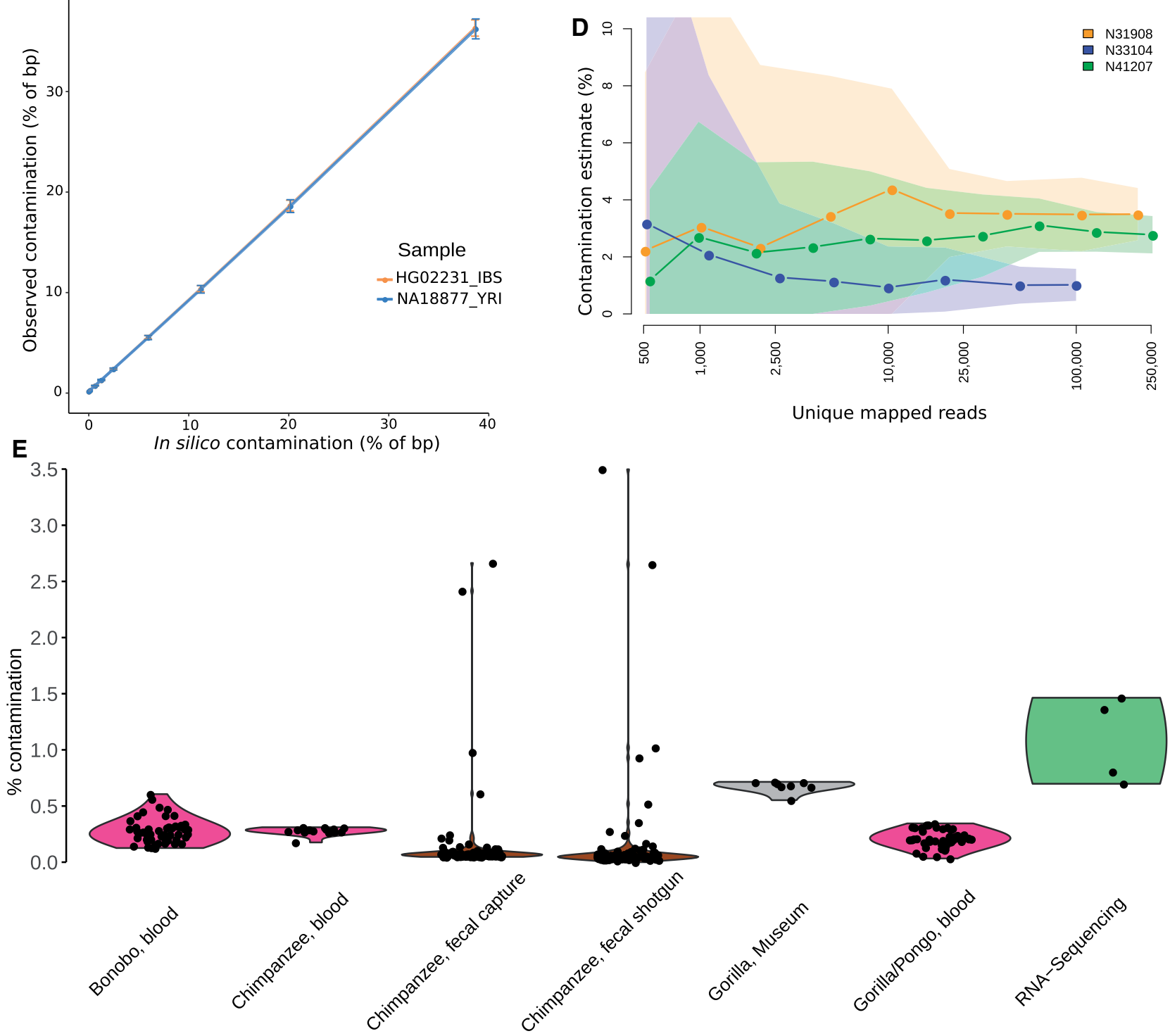

FIG. 1.-Summary of this study. (A) Schematic tree of the great ape species, with approximate divergence times (Besenbacher et al. 2019). (B) Schematic representation of the method. (C) Performance of detection of in silico-contamination in a gradient from $\sim 0.1$ to $39 \%$, point estimate \pm one standard deviation. (D) Performance when downsampling sequencing data from fecal samples with $1-3.5 \%$ of human contamination. Point estimates \pm one standard deviation. (E) Contamination estimates of blood samples for sequencing libraries from all species (violet; bonobo $N=52$, chimpanzee $N=15$; gorilla/orangutan $N=47$ ), fecal samples before and after capture (brown; $N=109$, without sample $N 42003$ which has high levels of non-great ape contamination), museum samples (grey; $N=8$ ) and RNA-sequencing data (green; $N=4$ ). 
the species combination. This is a consequence of the shared ancestry between humans and the other clades, where, for example, some of the chimpanzee-diagnostic alleles are the same in humans and gorillas, and other the same in chimpanzees and gorillas. This demonstrates that the test is cladespecific, and large amounts of reads that do not carry clade-specific alleles will be detected when a different primate species are present.

\section{Application to Other Sample Types}

We first applied the test to blood samples from all great ape species, which are generally expected to contain at most small amounts of human contamination. For 67 randomly chosen sequencing libraries from seven chimpanzees and four bonobo individuals (Prüfer et al. 2012), we found an average of $0.28 \%(0.13-0.61 \%)$ of reads that are putatively due to human contamination (fig. 1E). Four tissue samples from chimpanzees (White et al. 2019) show low estimates of contamination (0.03-0.067\%), as expected for samples likely not containing true contamination. Similar results are obtained for four libraries from gorilla $(0.033-0.159 \%$, on average $0.075 \%)$ and 43 libraries from orangutan $(0.08-0.35 \%$, on average 0.22\%) blood samples (Locke et al. 2011; Besenbacher et al. 2019). We conclude that traces of putative human contamination are observed, if at all, only at very small amounts in sequencing data from great ape blood samples. These estimates are conservative, since sequencing errors, mapping reference bias, and variation in these individuals may contribute to these numbers, especially considering that error rates of these sequencing technologies were decreasing after the publication of some of these studies (Locke et al. 2011; Prüfer et al. 2012). We also note that results for data mapped to hg19 and hg38 are almost identical (supplementary table S6, Supplementary Material online).

We then applied the contamination test to noninvasive samples which usually contain small amounts of host DNA and may require target hybridization methods to obtain sufficient data (Hernandez-Rodriguez et al. 2017; Fontsere et al. 2020). We applied our method to shotgun and exome capture sequencing data that were obtained from the same 109 sequencing libraries from chimpanzee fecal samples (White et al. 2019). We found an average of $0.35 \%(0-24.6 \%)$ human contamination for the precapture (shotgun) and $0.32 \%$ $(0.05-21 \%)$ human contamination in the postcapture (enriched) sequencing data (supplementary table S6, Supplementary Material online, fig. 1E), with strong correlation for the same samples $\left(r=0.99, P\right.$ value $\left.<2.2 \times 10^{-16}\right)$. We find one sample with an estimate of $24.6 \%$ and three more samples with more than $1 \%$ of human contamination (supplementary table S6, Supplementary Material online). In the case of fecal samples collected from the field that may contain other mammalian DNA than the target species through diet or misidentification, it is advisable to perform a competitive mapping of sequences when large amounts of contamination are detected. This will help to determine the species of origin, for example using BBSplit (https://sourceforge.net/projects/bbmap/, last accessed May 27, 2021) with a reference panel of great apes, and possibly other primate species living in the same habitat. We applied this method to these four samples (N42003_Shotgun1, N31908_Shotgun1, N33104_Shotgun1, and N41207_Shotgun1), and find that the main contaminant in one sample is most likely another primate rather than human (supplementary table S7, Supplementary Material online). It is known that chimpanzees hunt other primates (Boesch and Boesch 1989), and DNA from primate prey can persist in the feces. We conclude that the design of the contamination test presented here is able to identify reads carrying mutations that differ from the target species, even if these are not human-specific. When applying BBsplit method to in silico-contaminated samples, we confirm humans as the source of the contaminationalthough with less precision regarding the amount of contamination when compared to our method - while the majority of unambiguously mapped sequences align to the target species (supplementary table S7, Supplementary Material online).

Our analysis shows that DNA extracts/libraries from fecal samples are occasionally contaminated, and may need to be removed from certain downstream analyses. Hence, it is advisable to perform a contamination test for sequencing data from this type of sample, comparable to ancient and historical samples. We assessed the power to detect human contamination with very shallow sequencing, by downsampling the sequencing reads of the three fecal samples from White et al. (N31908_Shotgun1, N33104_Shotgun1, and N41207_Shotgun1) with 1-3.5\% human contamination. We downsampled these in several steps down to $\sim 1,000$ production reads (supplementary table S8, Supplementary Material online), and calculated the estimated amount of human contamination. These results (fig. 1D) confirm that our method is robust in confidently detecting human contamination even in the case of very shallow sequencing, as low as $\sim 1,000$ reads aligned to the human reference genome, although with a high standard deviation. In the case of fecal samples with around $5 \%$ of estimated host DNA, this could be as little as $\sim 20,000$ production reads, making the test applicable to shallow data from an initial screening procedure (Fontsere et al. 2020).

We also applied the test to published sequencing data from eight museum bone samples from gorillas (van der Valk et al. 2019). Here, we find an estimated contamination of on average $0.68 \%(0.55-0.72 \%)$, which is slightly lower than the reported estimates which were based on mitochondrial diagnostic loci (0.28-1.67\%, on average 1\%), and slightly higher than estimates for blood samples, as expected for museum specimens that have been handled by humans. Contamination estimates from mitochondrial and nuclear loci from the same sample have been found to not be identical in 
hominin samples (Prüfer et al. 2014), and at shallow sequencing coverage, a small number of reads would overlap with mitochondrial diagnostic loci. Still, the differences between these methods are minor, and results on data mapped to hg19 and hg38 are almost identical (supplementary table S6, Supplementary Material online), as is the case for blood samples. Finally, we performed the contamination test on RNA-sequencing data from great ape tissue samples (Brawand et al. 2011), mapped using tophat2 (Kim et al. 2013). We find slightly higher amounts of contamination (supplementary table S6, Supplementary Material online), either due to real contamination in the samples or to higher error rates and mapping bias in transcriptome data compared to genome sequencing data.

\section{Supplementary Material}

Supplementary data are available at Genome Biology and Evolution online.

\section{Method Availability}

The contamination test script including documentation is publicly available on GitHub: https://github.com/kuhlwilm/ HuConTest (last accessed May 27, 2021). Files with the diagnostic positions are publicly available on FigShare (doi: 10.6084/m9.figshare.14237834).

\section{Data Availability}

There are no new data associated with this article.

\section{Acknowledgments}

Wethank Marc de Manuel for help with data preparation. M.K. is supported by "la Caixa" Foundation (ID 100010434), fellowship code LCF/BQ/PR19/11700002. M.A.E. is supported by an FPI (Formación de Personal Investigador) PRE2018-083966 from Ministerio de Ciencia, Universidades e Investigación. T.M.-B is supported by funding from the European Research Council (ERC) under the European Union's Horizon 2020 research and innovation programme (grant agreement No. 864203), BFU2017-86471-P (MINECO/FEDER, UE), "Unidad de Excelencia María de Maeztu", funded by the AEl (CEX2018-000792-M), Howard Hughes International Early Career, Obra Social "La Caixa" and Secretaria d'Universitats i Recerca and CERCA Programme del Departament d'Economia i Coneixement de la Generalitat de Catalunya (GRC 2017 SGR 880).

\section{References}

Auton A, 1000 Genomes Project Consortium, et al. 2015. A global reference for human genetic variation. Nature 526(7571): 68-74.
Besenbacher S, Hvilsom C, Marques-Bonet T, Mailund T, Schierup MH. 2019. Direct estimation of mutations in great apes reconciles phylogenetic dating. Nat Ecol Evol. 32(2): 86-292.

Boesch C, Boesch H. 1989. Hunting behavior of wild chimpanzees in the Taï National Park. Am J Phys Anthropol. 78(4): 547-573.

Brawand D, et al. 2011. The evolution of gene expression levels in mammalian organs. Nature 478(7369):343-348.

Fontsere $C$, et al. 2020. Maximizing the acquisition of unique reads in noninvasive capture sequencing experiments. Mol Ecol Resour (21):745761. doi: 10.1111/1755-0998.13300.

Hernandez-Rodriguez J, et al. 2017. The impact of endogenous content, replicates and pooling on genome capture from faecal samples. Mol Ecol Resour (18):319-333. doi: 10.1111/1755-0998.12728.

Kaessmann H, Pääbo S. 2002. The genetical history of humans and the great apes. J Intern Med. 251(1): 1-18.

Kim D, et al. 2013. TopHat2: accurate alignment of transcriptomes in the presence of insertions, deletions and gene fusions. Genome Biol. 14(4): R36.

Kuhlwilm M, et al. 2016. Evolution and demography of the great apes. Curr Opin Genet Dev (41):124-129. doi: 10.1016/j.gde.2016.09.005.

Lawrence M, Gentleman R, Carey V. 2009. rtracklayer: an R package for interfacing with genome browsers. Bioinformatics 25(14): 1841-1842.

Li H, 1000 Genome Project Data Processing Subgroup, et al. 2009. The Sequence Alignment/Map format and SAMtools. Bioinformatics 25(16): 2078-2079.

Li H, Durbin R. 2009. Fast and accurate short read alignment with Burrows-Wheeler transform. Bioinformatics 25(14): 1754-1760.

Locke DP, et al. 2011. Comparative and demographic analysis of orangutan genomes. Nature 469(7331): 529-533.

Mallick S, et al. 2016. The Simons genome diversity project: 300 genomes from 142 diverse populations. Nature 538(7624): 201-206.

De Manuel M, et al. 2016. Chimpanzee genomic diversity reveals ancient admixture with bonobos. Science (80-) 354(6311): 477-481.

McKenna A, et al. 2010. The Genome Analysis Toolkit: MapReduce framework for analyzing next-generation DNA sequencing data. Genome Res. 20(9): 1297-1303.

Meyer M, et al. 2016. Nuclear DNA sequences from the Middle Pleistocene Sima de los Huesos hominins. Nature 531(7595): 504-515.

Nater A, et al. 2017. Morphometric, behavioral, and genomic evidence for a new orangutan species. Curr Biol. 27(22): 3487-3498.e10.

Peyrégne S, Prüfer K. 2020. Present-day DNA contamination in ancient DNA datasets. BioEssays 42(9): 2000081.

Prado-Martinez J, et al. 2013. Great ape genetic diversity and population history. Nature 499(7459): 471-475.

Prüfer K, et al. 2012. The bonobo genome compared with the chimpanzee and human genomes. Nature 486(7404): 527-531.

Prüfer K, et al. 2014. The complete genome sequence of a Neanderthal from the Altai Mountains. Nature 505(7481): 43-49.

R Core Team. 2015. R: a language and environment for statistical computing. http://www.r-project.org/. Accessed May 27, 2021.

Schubert M, Lindgreen S, Orlando L. 2016. AdapterRemoval v2: rapid adapter trimming, identification, and read merging. BMC Res Notes. 9:88.

van der Valk T, Díez-del-Molino D, Marques-Bonet T, Guschanski K, Dalén L. 2019. Historical genomes reveal the genomic consequences of recent population decline in eastern gorillas. Curr Biol. 29(1): 165-170.e6.

White LC, et al. 2019. A roadmap for high-throughput sequencing studies of wild animal populations using noninvasive samples and hybridization capture. Mol Ecol Resour. 19(3): 609-622.

Xue $Y$, et al. 2015. Mountain gorilla genomes reveal the impact of longterm population decline and inbreeding. Science 348(6231): 242-245.

Associate editor: David Enard 